Available online @ https://jjem.jnnce.ac.in https:www.doi.org/10.37314/JJEM.2020.040102 Indexed in International Scientific Indiexing (ISI) Impact factor: 1.025 for 2018-19 Published on: 30 November 2020

\title{
Diabetic Retinopathy Detection by Image Processing Algorithms and Machine Learning Technique
}

\author{
JNN College of Engineering, Shimoga, India. \\ ayubkhan1998@gmail.com, balgi05anantraya@gmail.com, \\ chinthucm103@gmail.com,pramodkumars@jnnce.ac.in
}

Mohammed Ayub Khan, Anantraya Prabhakar Balgi, Chaithra C, Pramod Kumar S

\begin{abstract}
Diabetic disease is a persistent sickness caused by both absence of insulin production in body or incapacity to withstand insulin in the body. Due to diabetic disease it causes cardiac problems, vascular ailment and paralysis, kidney disorder. Neuropathy and diabetic eye disease disorder called as Diabetic Retinopathy. Diabetic disease for an extended time damages nerves of retina and thereby reducing vision capacity of a person and leading to diabetic retinopathy. Here the classification is done into three stages. They are Normal eye, Diabetic Retinopathy eye and Age-related Macular Degeneration (AMD). Diabetic retinopathy finding in fundus image is detected through image processing and device mastering strategies. Here we use Support Vector Machine (SVM) for purpose of classification which is most suitable in this work.
\end{abstract}

Keywords: Retinopathy, Segmentation, Image Processing

\section{INTRODUCTION}

Diabetic disease is a persistent ailment which cause harm to retina. This is due to loss of sugar level in body or incapability of frame cells in response to fluid in the frame. Nerves in eye getting into the retina from the optic disc are broken which bring about lack of vision. Initial degree suggests as one, however with time and shortage of diabetes increment may cause very much loss of imaginative and prescient. In this quick paced presence of diabetes is evaluated to ascend from 415 million out of 2015 to 642 million out of 2040 . Diabetic retinopathy causes lack of sight in 1.8 million from 37 million human beings. Early stage of this disorder called non proliferative diabetic retinopathy and is shows symptoms of micro-aneurysms, exudates and haemorrhages. Micro-aneurysm are small crimson spots taking place within the retina due to swellings in capillaries. Exudates are white or yellow deposits precipitated leaking fluid from the capillary. Haemorrhages are red spots because of capillary rupturing and micro aneurysms. In future degree of diabetic retinopathy referred to as proliferative diabetic retinopathy is characterized with the aid of immoderate boom of new nerves within the retina. Exuding of nerves cause lack of vision as shone in figure 1.

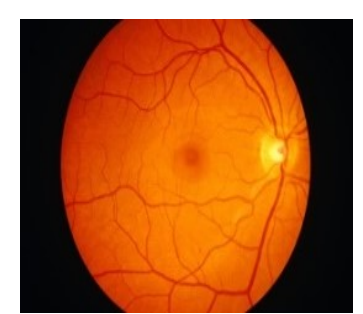

(a)

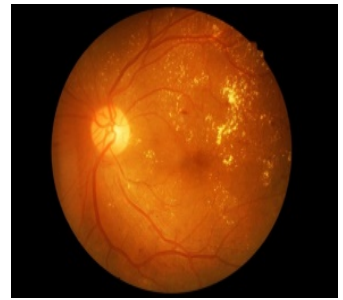

(b)
Figure 1: Retina, (a) Healthy Retina b) Diabetic Retina.

This diabetic eye disease is a hassle of diabetes and cause purpose of blindness. It 
happens when diabetes harms the small blood vessels inside the retina, the light touchy tissue behind sight.

\section{Literature survey}

As there are advancements in care and safety for eye vision. As such a whole lot of studies have been taken for location on automated diagnosis of diabetic retinopathy using some of advanced and greater correct and exact techniques. To point out a number of important strategies, advanced as consistent with the research worked on a method on automated detection and type of vascular problems in this diabetic eye.

The system allows incredible to facilitate diagnosis by using the ophthalmologists, properly advanced content primarily based image retrieval approach [1]. This method facilitates in verifying the hypothesis that retinal pathology can be identified and quantified. It uses visually similar retinal photographs from an available image archive and the issues in detecting nerves in retinal photographs [2]. In this study, the concept of matched clear out for detection of indicators to detect piecewise linear segments of blood vessels in retinal images considered [3, 4]. This cause creation of one-of-a-kind templates for looking vessel segments along all valuable guidelines. The research result in the detection of most of the features inclusive of nerves, exudates and optic disk and greater important with accurately using of image operating algorithms [5]. The research revealed the use of back-propagation neural network, the Bayesian neural network, the probabilistic neural community and the aid vector device, all applied to develop class fashions for finding Ischemic Heart Disease (IHD) sufferers [6, 7]. Their studies made use of a guide vector device with Gaussian radial basis characteristic as a classifier. As such they might perceive tough exudates from digital retinal images used a support vector machine for the detection of diabetic eye degrees.

\section{Proposed system}

This work provides a quick approach which fastens up the finding and categorizes disorder in the diabetic eye disease. A lower strength microscope named ophthalmoscope or the fundus digital camera is connected with a virtual digital camera captures the image of interior surface of the eye which incorporates retina, optic discs, macula and the blood vessels. The pictures are usually acquired from the posterior pole's view along with the optic disc and macula. Images had the resolution of $1280 \times 1024$ or $700 \times 605$ Pixels.

The proposed device classifies the images into classes together with normal, diabetic eye and AMD. For evaluation dataset of thirty-six images had been considered. The proposed machine converts the colour coordinate photograph obtained from the fundus digital device into gray image. Adaptive histogram equalization applied to the image that improvises texture. Followed by means of flexible histogram levelling, disturbance in the image is removed by using the matched filter response.

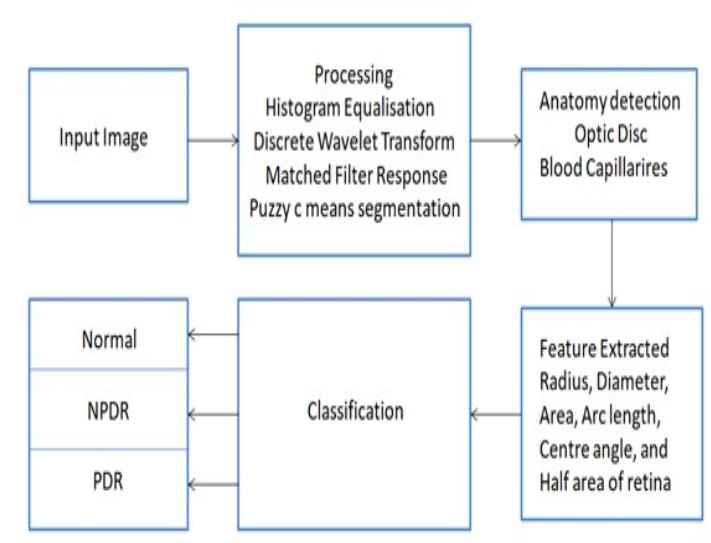

Figure 2: Flow Diagram of Proposed system

In next step of the operation involves the clustering by using K-means method which makes the blood vessels of the image fairly seen. After operating on image, functions such features inclusive of radius, diameter, area, arc length, centre angle and half region are 
calculated for every image. Features extracted are exceeded directly to the machines getting to know some of the useful information's along with vector gadget and other techniques for classifying the image into normal, diabetic eye and AMD. The proposed procedure shown in figure 2.

\section{Explanation of working of the system}

\subsection{Pre-Processing Stage}

\subsubsection{Conversion of Colour Space}

The context of converting an image for representing in one coloration space to other suitable shade space, the intention being to make the translated image look as comparable as valuable to the authentic.

\subsubsection{Edge Zero Padding}

This is a way that output pixels on the right fringe of the image could be suffering from pixels on the left facet, zero-padding allows area for this wrapround to arise without contaminating real output pixels.

\subsubsection{Median Filtering}

The median filter is a lateral essential filtering method, frequently used to take away disturbance from an image or sign. Such disturbance decrement is a typical preoperating step to improve the results of later procedure.

\subsubsection{Adaptive Histogram Equalization}

The adaptive histogram equalization is used to improve intensity in image. Images are rarely defected by worse illumination and are dark. This happens usually in medical images or the image acquired in less light situations. The proposed procedure shown in the figure 3.

\subsection{Segmentation Stage}

\subsubsection{Class Segmentation}

The aim of segmentation is to simplify and alternate the illustration of an image into entity that is greater significant and less difficult to investigate. Image segmentation is usually used to find border and edges (traces etc.) in snap shots.

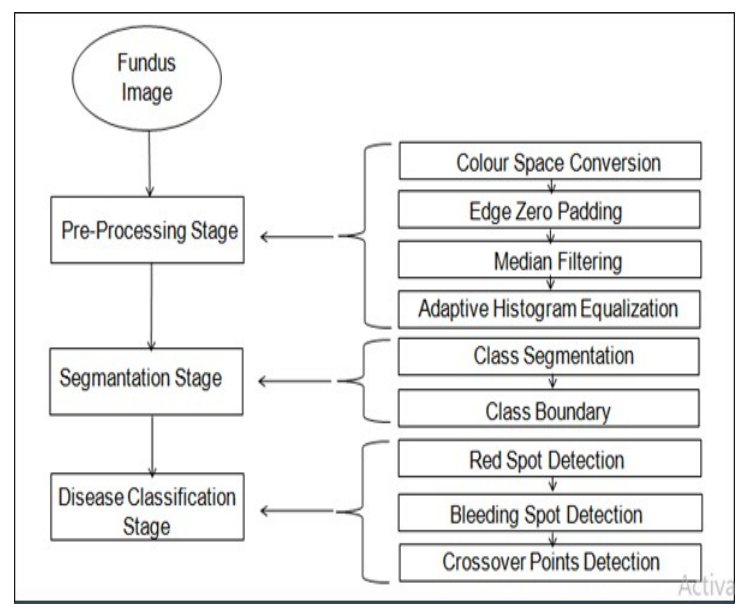

Figure 3: Block diagram of methodology.

\subsubsection{Class Boundary}

Class boundaries are the numbers used to split training. Class boundary is the midpoint of the higher magnificence limit of one class and the decrease class limit of the following elegance. Each class consequently has a higher and a decrease elegance boundary.

\subsection{Disease Classification Stage}

\subsubsection{Red Spot Detection}

Early stage signal of diabetic retinopathy is called as crimson lesions. Crimson lesions include microaneurysms and haemorrhages. Unpigmented lesions consist of damages and cotton-wool points. These are signals of non- proliferative diabetic eye disease.

\subsubsection{Bleeding Spot Detection}

It is a brand new, a typical blood vessel that shape in the lower back of eye of the human beings with diabetes.

\subsubsection{Crossover Points Detection}

This entails as it should be searching over-crossed points or layer convergence spots in a fundus image. 


\section{Methods for proposed methodology}

\subsection{Databases}

Freely getting databases which include Drive, Diaretdb1 and Messidor are used. The proposed procedure as shown in the figure 4.

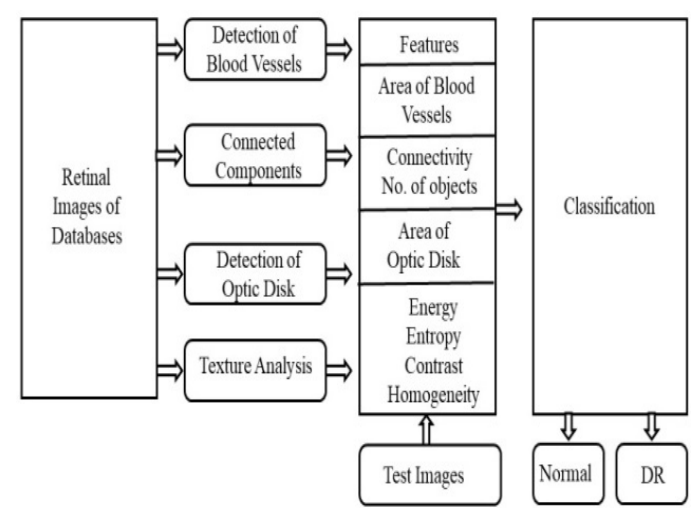

Figure 4: Block Diagram of Proposed Methodology.

\subsubsection{Sectionalisation of Retinal Shape}

Finding of blood vessels, it is used for reinforcing the retinal image. Finding of damages and micro aneurysms morphological operators are used for the finding of exudates.

\subsubsection{Texture Analysis}

It gives statistics, approximately the position of aspect pixels and their connection with the encircling pixels.

\subsubsection{Feature Extraction}

The function vector used for category received from sectionalizing of retinal shapes and quality analysis.

\subsubsection{Classification}

Based on the SVM classifier we can classify images of retina into 3 , by the use of feature extraction and they are:
a. Normal Eye.
b. Diabetic Retinopathy
c. AMD

\section{Observation and analysis}

The Observation and analysis consist of following steps to do:
a. Pre-processing.
b. Segmentation.
c. Feature Extraction.
d. Feature Matching.
e. Feature Detection.
f. Classification.

\subsection{Pre-processing}

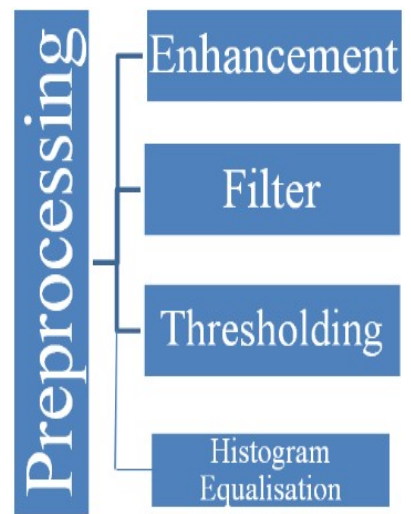

Figure 5: Pre-processing stage

The retinal fundus images are generally used for detection and evaluation of diabetic eye disease. The pre-processing of raw retinal fundus snap shots is done by the usage of extraction of green channel, image enhancement and resigning. The given retinal image is pre-processed to obtain the vicinity of interest to extract the function with the help of filter. The proposed procedure shown in figure 5.

Initially, the images are resized and sampled into the desired size $200 \times 200$. Then filtering strategies like gaussian filtering, grey scale conversion are hired to transform the image into Gray scale, then to decrease disturbance in image one by one, as shown in figure 6 .

In this pre-processing, an algorithm is used called as Otsu method. It is used to carry out automated image thresholding. In this only shape, the set of rules returns a single depth threshold that separate pixels into two training and foreground.

Initial step is concerned the pre-processing and resizing the images. Before giving into the architecture for category, it is an one colour 
image which is used to show the microaneurysms, and nerves within the fundus images and flatten the images in unmannered dimension for operating similarly. The fundus snap shots are received from one kind of statistics sets and are taken under exceptional digital camera with various area of view, non- readability, lossy, comparison and sizes of photographs one of a kind. In this step evaluation adjustment, flipping images, brightness adjustments are made. Then the images are resized and sampled into the required length.

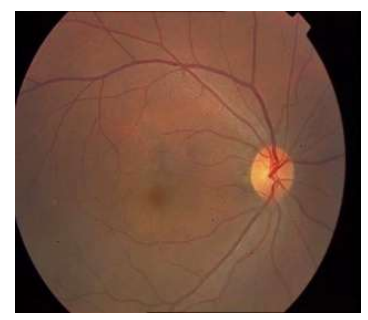

(a) Initial Image

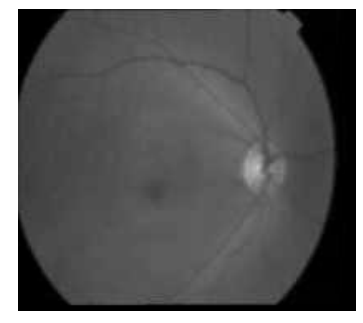

(b) Pre-processed Image Figure 6: Pre-processing.
The median filter is used to remove disturbance from an image, the median filter is for lateral digital filtering technique, such disturbance discount is an ordinary pre-processing step to better the outcomes of later procedure.

\subsection{Segmentation}

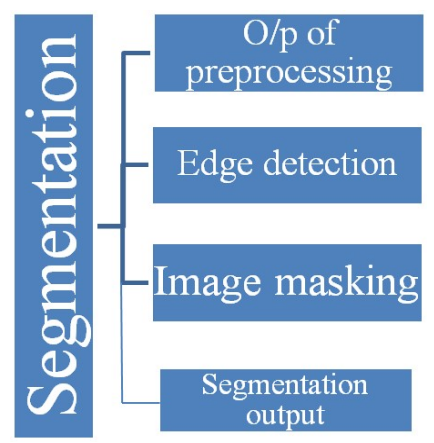

Figure7: Segmentation stage

It is the method of partitioning the digital picture into more than one segments (set of pels). Segmentation is to simplify and trade the illustration into image into something easier, makes extra significant and less complicate for analysis. There are distinctive techniques present in this segmentation. The proposed procedure shown in figure 7.

In the segmentation, degree is taken as the output image of pre-processing as it enters into image for segmentation.

The set of rules used for the segmentation stage is K-means algorithm. To discover edges, canny side detection approach is used. Image overlaying is carried out via pixel by using pixel as shown in figure 8 .

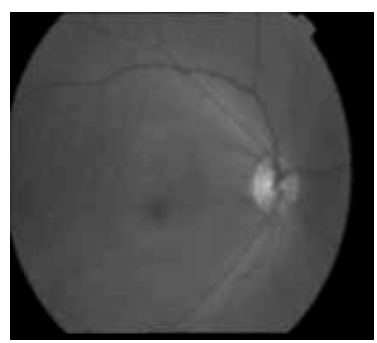

(a)

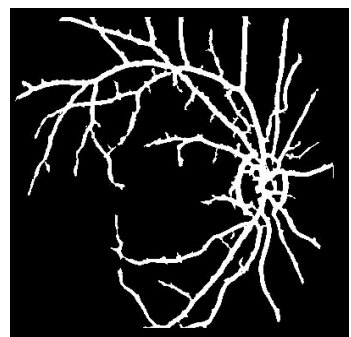

(b)
Figure 8: Image Segmentation (a) Output of pre-processing. (b) Image Segmentation.

\subsection{Feature Extraction}

In feature extraction, convert the input data into set of features. Here following methods can be implemented, that is by Gray-Level Co-Occurrence Matrix (GLCM) based texture features as shown in figure 9.

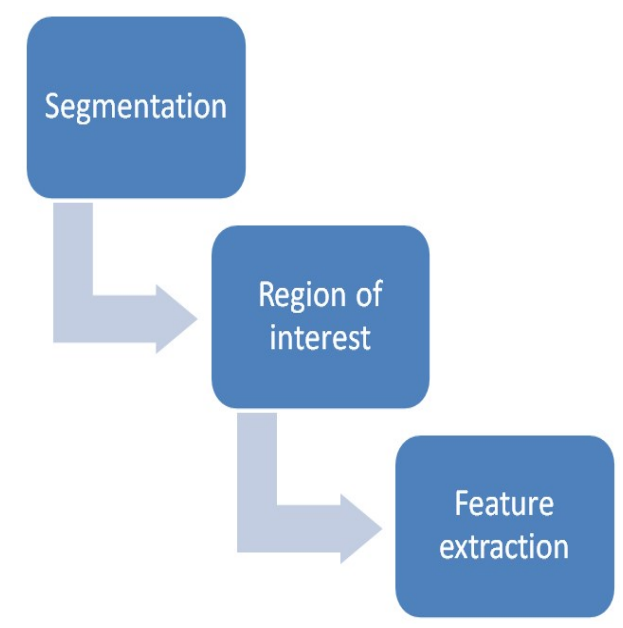

Figure 9: Feature Extraction stage. 


\subsection{Feature Matching}

The registered image and new retinal image of identical person can be matched by use of optic disc vascular bifurcation and distribution as shown in figure 10 .

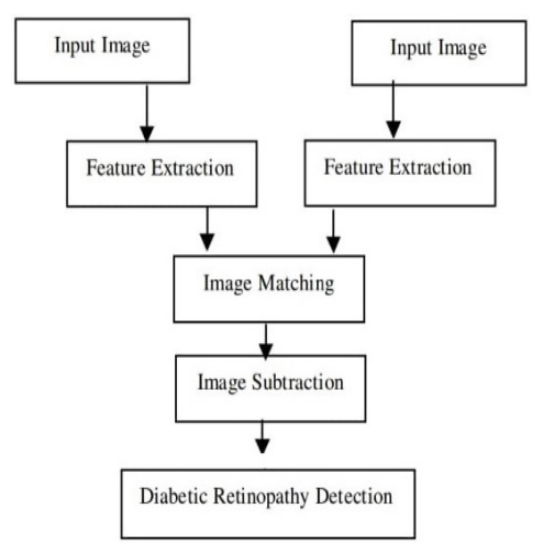

Figure 10: Feature Matching Stage.

\subsection{Feature Detection}

In detection level the processed image may be in comparison with the all the different processed images within the database. The functions like lesions exudates are compared.

Finding of exudates is fundamental requirement to diagnose the process of diabetic retinopathy. The optical disc removal is completed within the detection, early detection can probably reduce the threat of blindness.

\subsection{Classification}

SVM: A support vector system is a superintended machine learning version that uses category algorithms for 2-structure class problems. After giving an SVM version units of labelled training statistics.

In machine getting to know, assist-vector machines (SVMs, also support-vector networks) are superintended studying fashions with related gaining knowledge of algorithms that examine information used for class and regression evaluation.
Correlation: Correlation evaluation is used to quantify the degree to which two variables are related. Through the correlation evaluation, with one variable adjustment while the other one does. Correlation analysis affords a linear courting among two variables.

Erosion and Dilation: In erosion the image is processed based on shapes of various image objects. It is fashioned via structuring detail. It has matrix which contains 1 's and 0 's where 1 's is referred to as neighbourhood pixels. Here, the 'disk' structuring element is used to dilate and erode the image for vessel extraction. The mixture of dilation and erosion operations are completed on image with special structuring detail of radii.

In dilation, adding a pel to item edges primarily based on shaping detail. The prototype to discover output pel is the most of input pels community matrix.

LBP: Local Binary Pattern (LBP) is used to obtain much green quality which shows the pixels of an image by means of thresholding the community of every pixel and takes the output as a binary wide variety. It became first described in 1994 and has on the grounds that been found to be an effective feature for texture type.

LBP is a texture descriptor for retinal images. The texture of the retina historical past is analysed by LBP, and is used to differentiate healthy and the diseased. The maximum crucial capabilities of the LBP algorithm are proof against Gray degree modifications and computational simplicity which may be used in real-time applications.

\section{Software details}

For implementation and verification MATLAB because for multi-purpose computing surroundings and user-friendly programming language advanced with the aid of MathWorks. It lets in many matrix functions, drawing of statistics and functions, 
implementation of algorithms, introduction of person interface, and managing with programs written in other languages.

\section{Advantages, disadvantages and application}

Advantages: Provides a very good framework for Diabetic Retinopathy. Various capabilities together with haemorrhages, microaneurysms, tough exudates and gentle exudates may be detected.

Disadvantages: Can only detect diabetic Retinopathy.

Application: Medical centres like hospital, anganwadi.

\section{Result}

\subsection{Result obtained for normal stage}

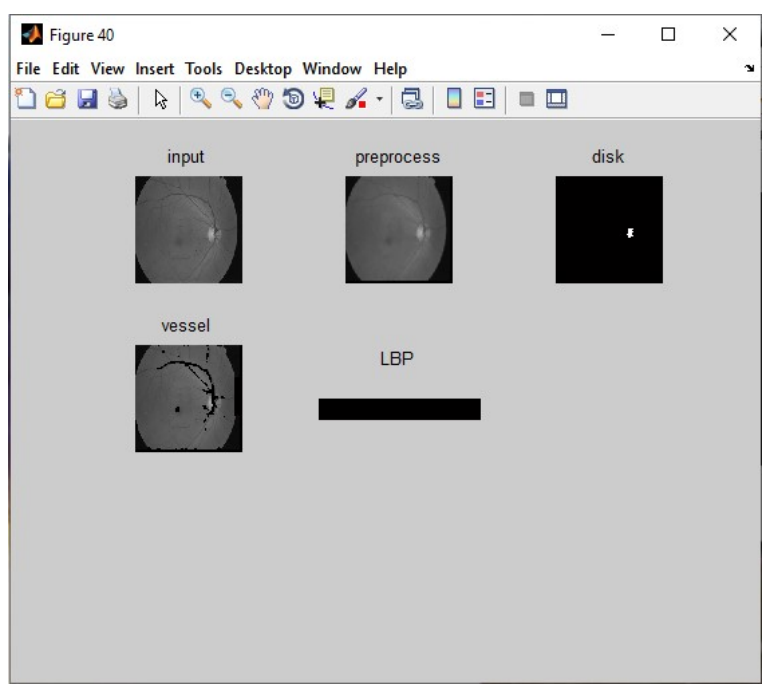

(a) Output image

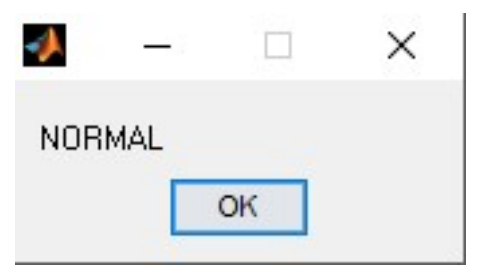

(b) Stage

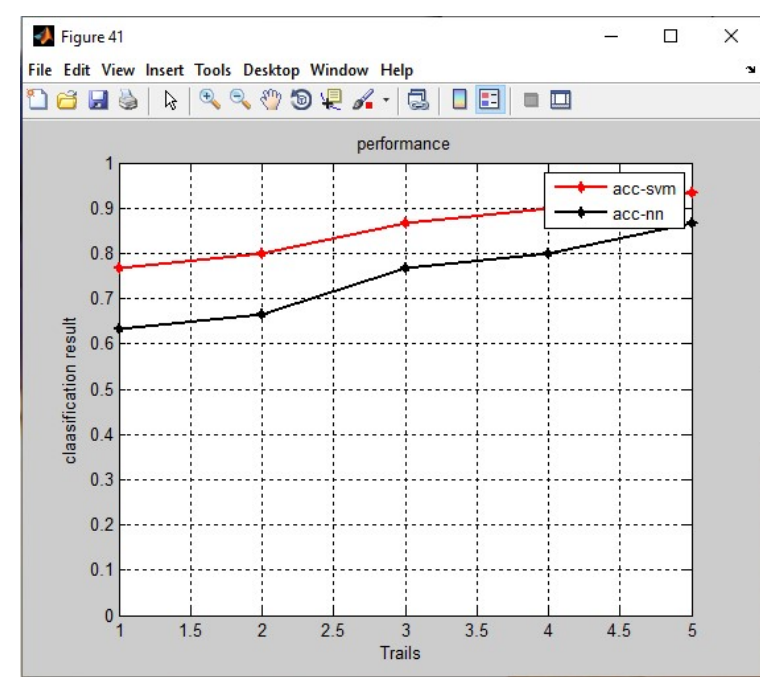

(c) Correlation

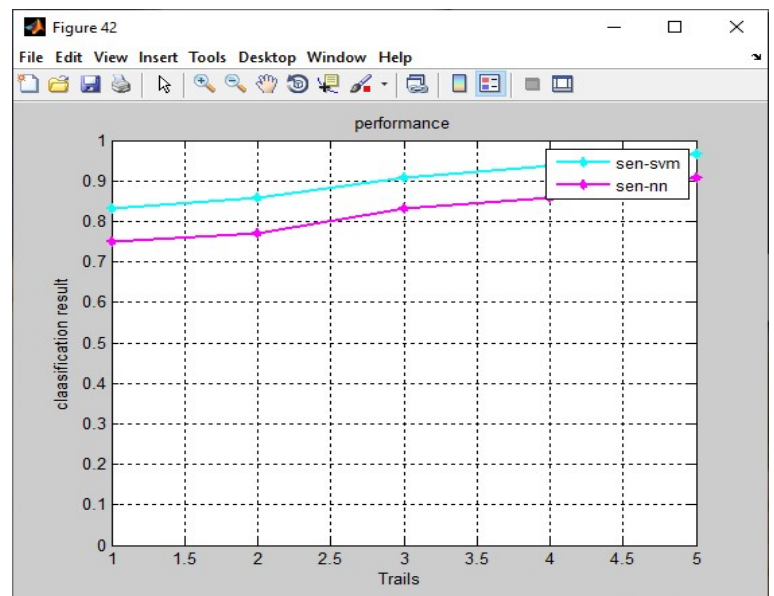

(d) Entropy

Aigure 43

File Edit View Insert Tools Desktop Window Help

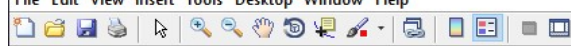

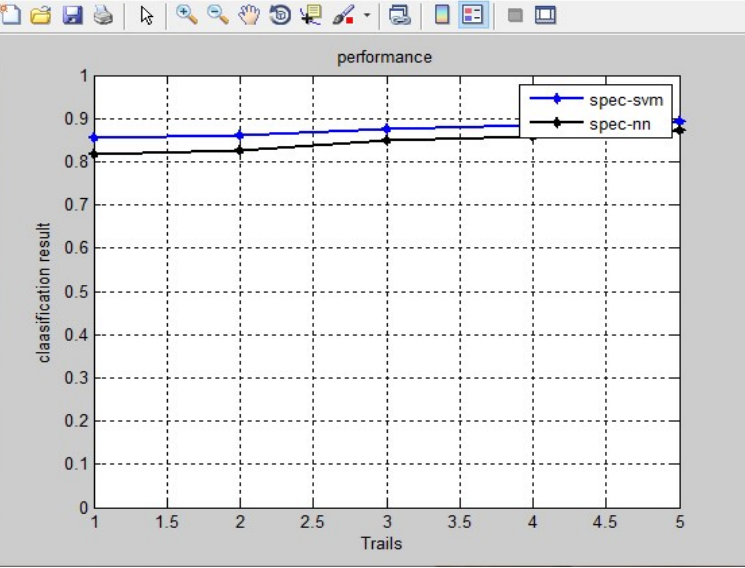

(e) LBP

Figure 11: Result obtained for normal stage describes that person has clear vision. 


\subsection{Result obtained for diabetic stage}

\section{Figure 40}

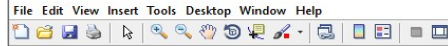
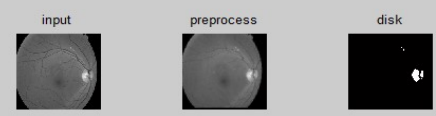

vessel
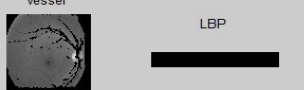

(a) Output image

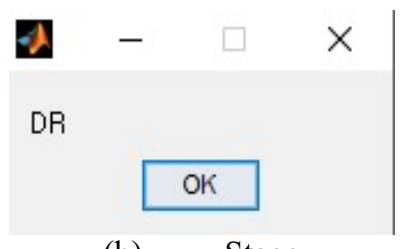

(b)

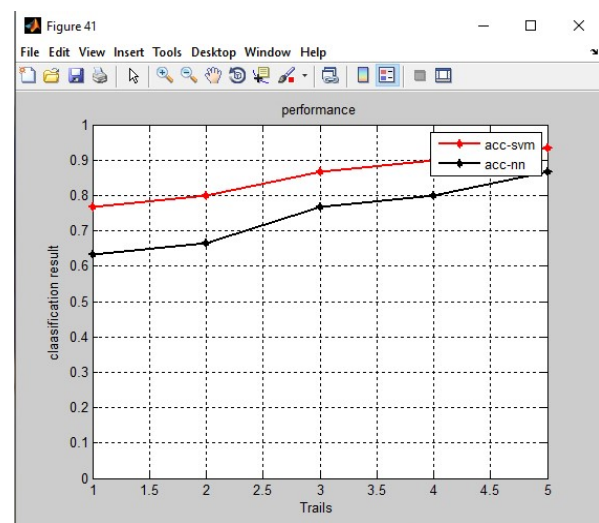

(c) Correlation

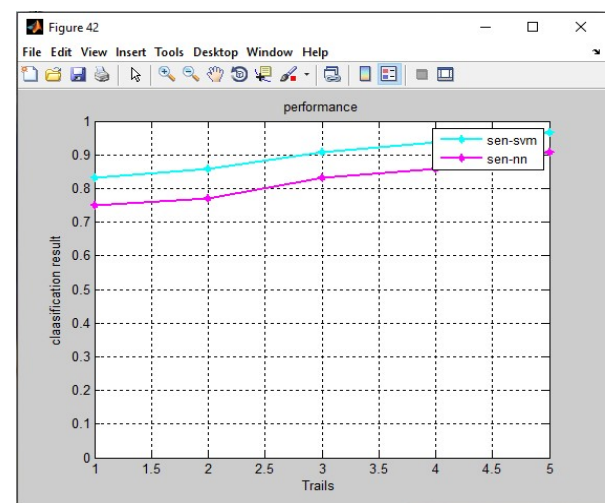

(d) Entropy

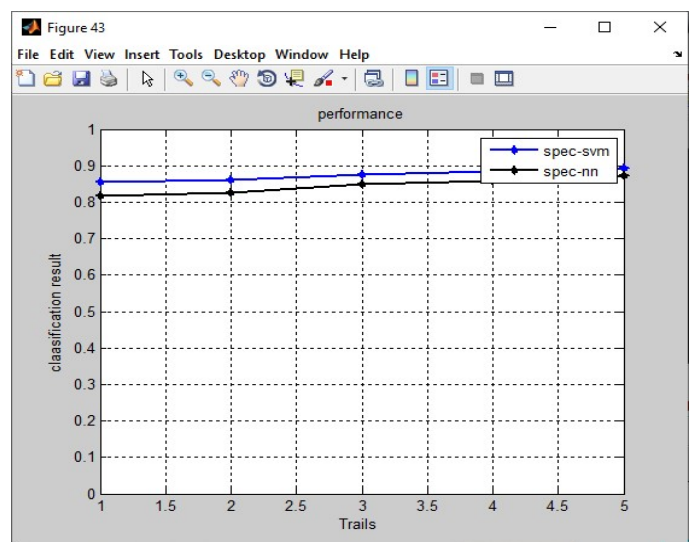

(e) LBP

Figure 12: Result obtained for diabetic stage describes that person has diabetes and can be cured by treatment

\subsection{Result obtained for AMD stage}

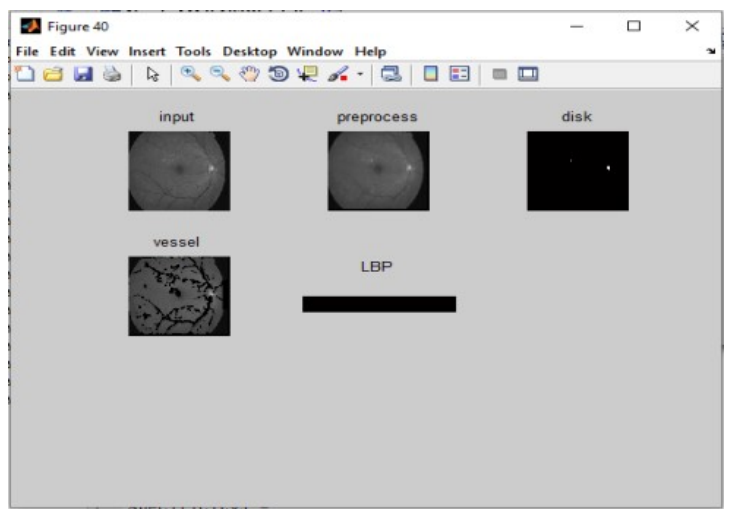

(a) Output image

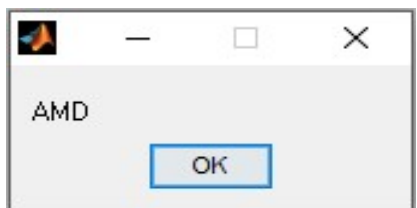

(b) Stage

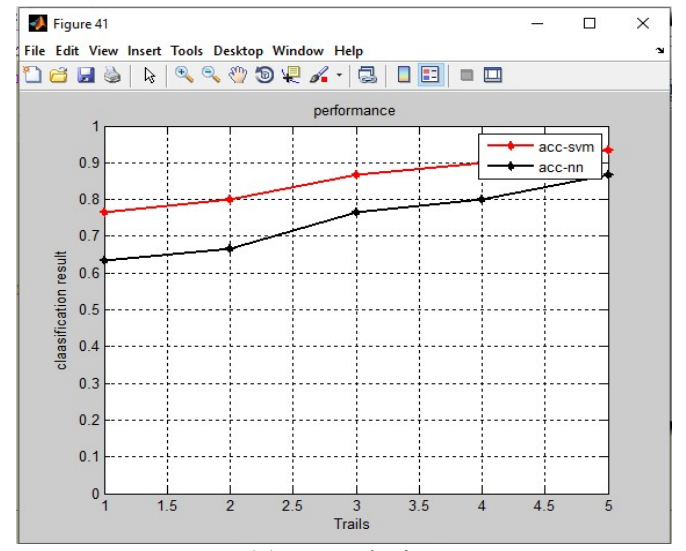

(c) Correlation 


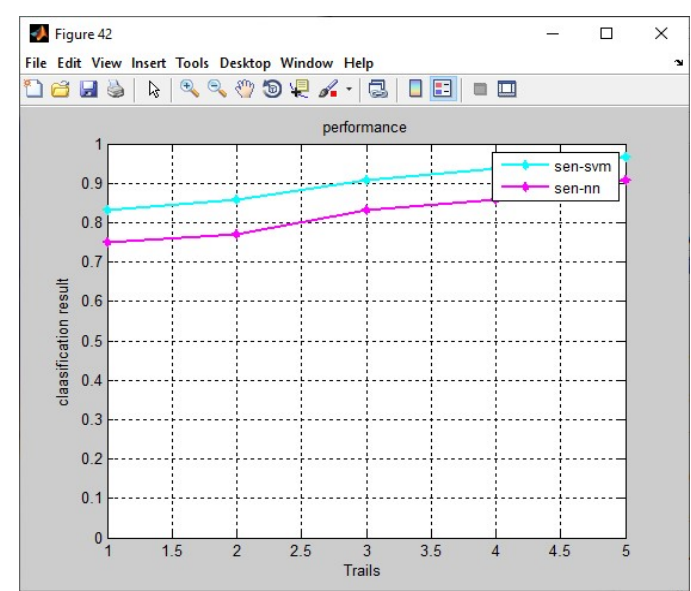

(d) Entropy

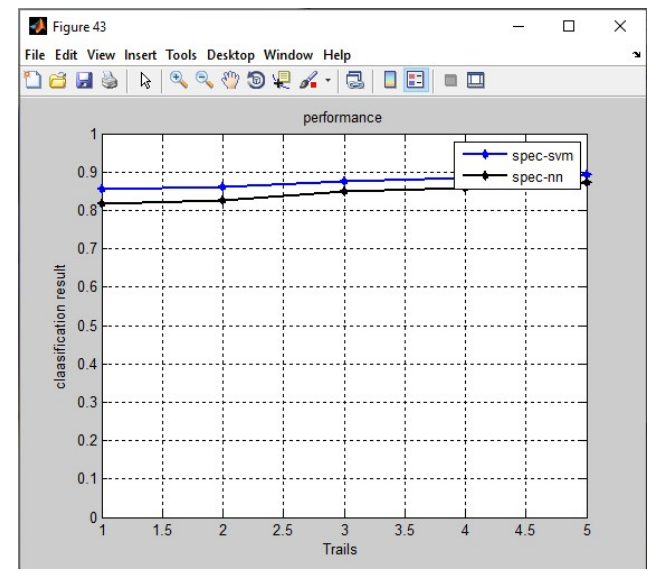

(e) LBP

Figure 13: Result obtained for AMD stage describes severe infection and vision loss.

\section{Conclusion}

An efficient procedure is proposed to extract features of retinal image. Extracted features will be subjected to processing retinopathy stage in retina image and then results will be classified using SVM Classifier.

\section{References}

1. Salman Sayed, Vandana Inamdar, Sangram Kapre, "Detection of Diabetic Retinopathy using Image Processing and Machine Learning", International Journal of Innovative Research in Science, Engineering and Technology, Vol. 6, Issue 1, January 2017, PP 99-107.

2. T Chandrakumar, "Classifying Diabetic Retinopathy using Deep Learning Architecture", International Journal of Engineering Research \& Technology (IJERT), 06-June-2016.

3. M Kamaladevi, S Sneha Rupa, T Sowmya, "Automatic Detection of Diabetic Retinopathy in Large Scale Retinal images", School of computing, SASTRA Deemed to be University, INDIA, 2018.

4. Kumar, S Pramod, and Mrityunjaya V Latte. "Lung parenchyma segmentation: fully automated and accurate approach for thoracic CT scan images." IETE Journal of Research 66, no. 3 (2020): 370-383.

5. Sirpa. M R, Vyshnavi G K P, Chandramoorthy M, Padmapriya B, "Efficient Detection of Retina Blood Vessels Using Proficient Morphological Algorithms", International Research Journal of Engineering and Technology (IRJET), Mar-2016.

6. R Subhashini, T N R Nithin, U M S Koushik, "Diabetic Retinopathy Detection using Image Processing", International Journal of Recent Technology and Engineering (IJRTE) , July 2019.

7. Kumar, S Pramod, and Mrityunjaya V Latte. "Fully automated segmentation of lung parenchyma using break and repair strategy." Journal of Intelligent Systems 28, no. 2 (2019): 275-289. 